\title{
A Nano-Printed Model of Interstitial Cancer Migration Reveals a Link Between Cell Deformability and Proliferation
}

\section{Supporting Information}

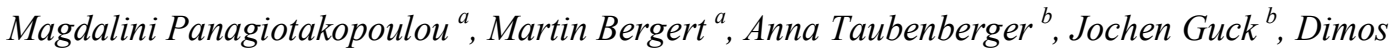

\author{
Poulikakos ${ }^{a^{*}}$, and Aldo Ferrari ${ }^{a^{*}}$.
}

a. ETH Zurich, Laboratory of Thermodynamics in Emerging Technologies, Sonneggstrasse 3, CH-8092 Zurich, Switzerland.

b. Biotec TU Dresden, Am Tatzberg 47-49, 01307 Dresden, Germany.

Keywords: Interstitial migration, cancer, pore penetration, cell cycle, chromatin condensation

* To whom correspondence should be addressed.

Dr. Aldo Ferrari - aferrari@ethz.ch

Prof. Dimos Poulikakos - dpoulikakos@ethz.ch

ETH Zurich

Department of Mechanical and Process Engineering

Sonneggstrasse 3, ML J 27.1

CH-8092 Zurich

SWITZERLAND

Phone: +41 446322588

Fax: +41 446321176 
$\underline{\text { Supplementary Figures and Videos }}$ 
HeLa Kyoto

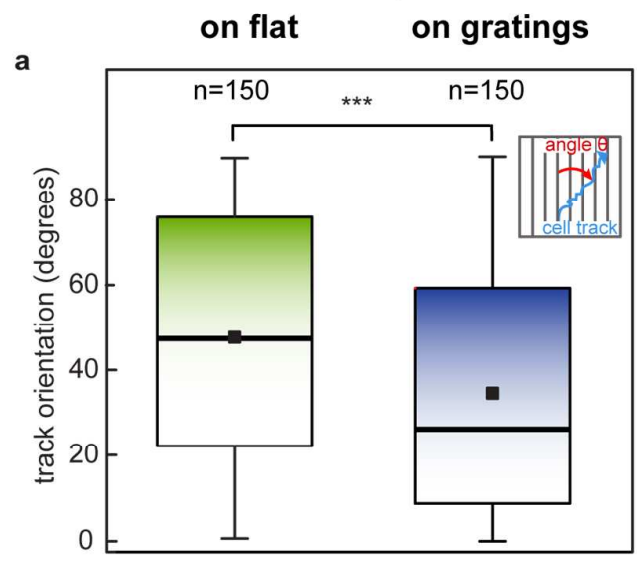

c

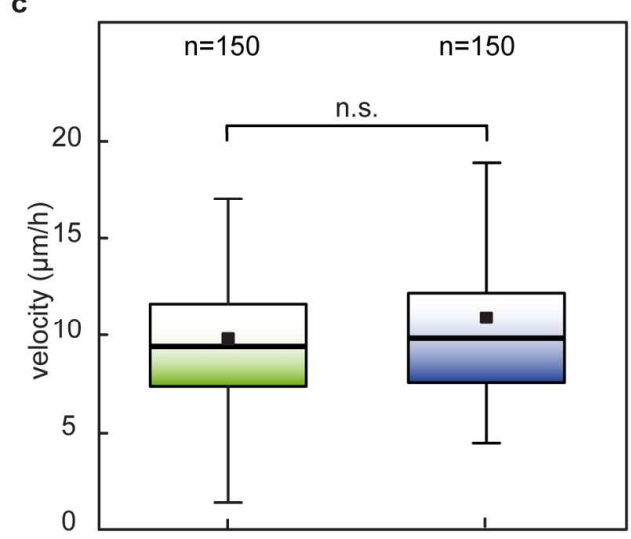

e
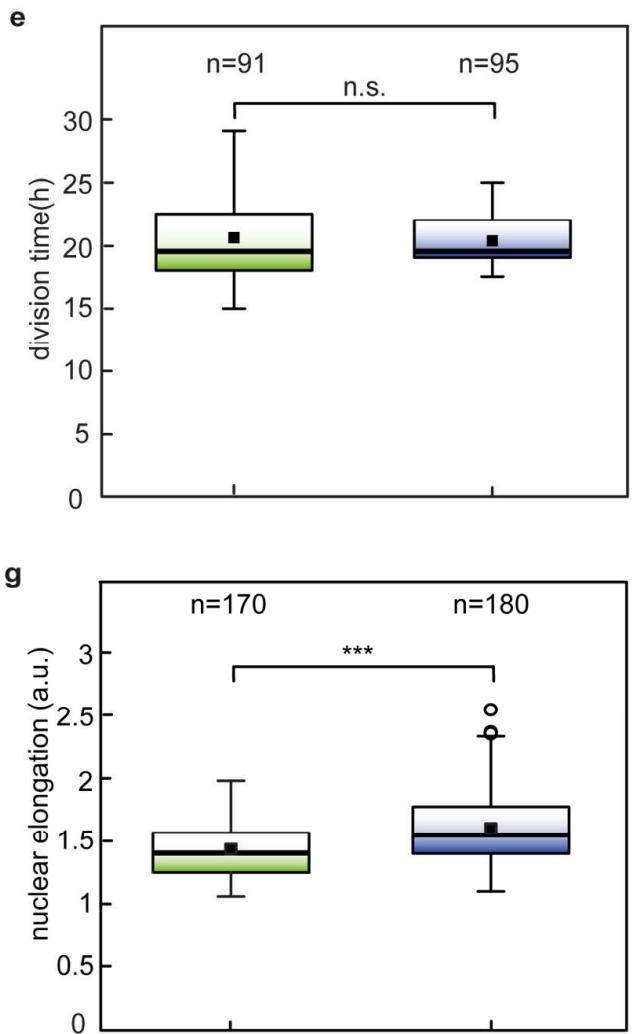

MCF10CA1a.cl1

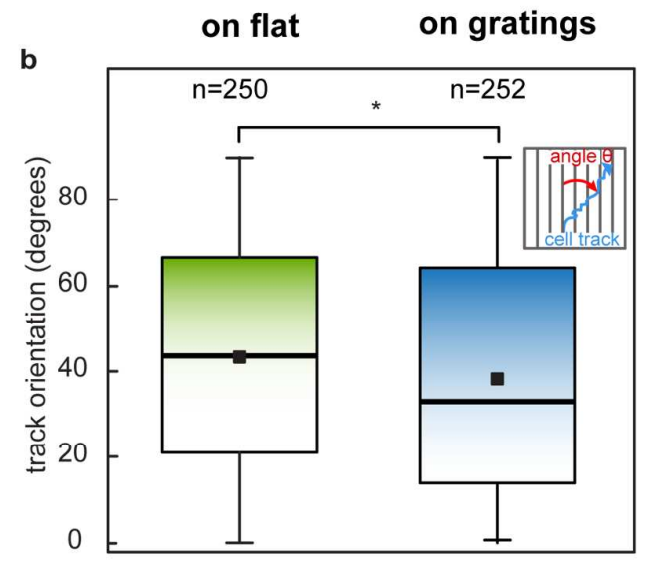

d
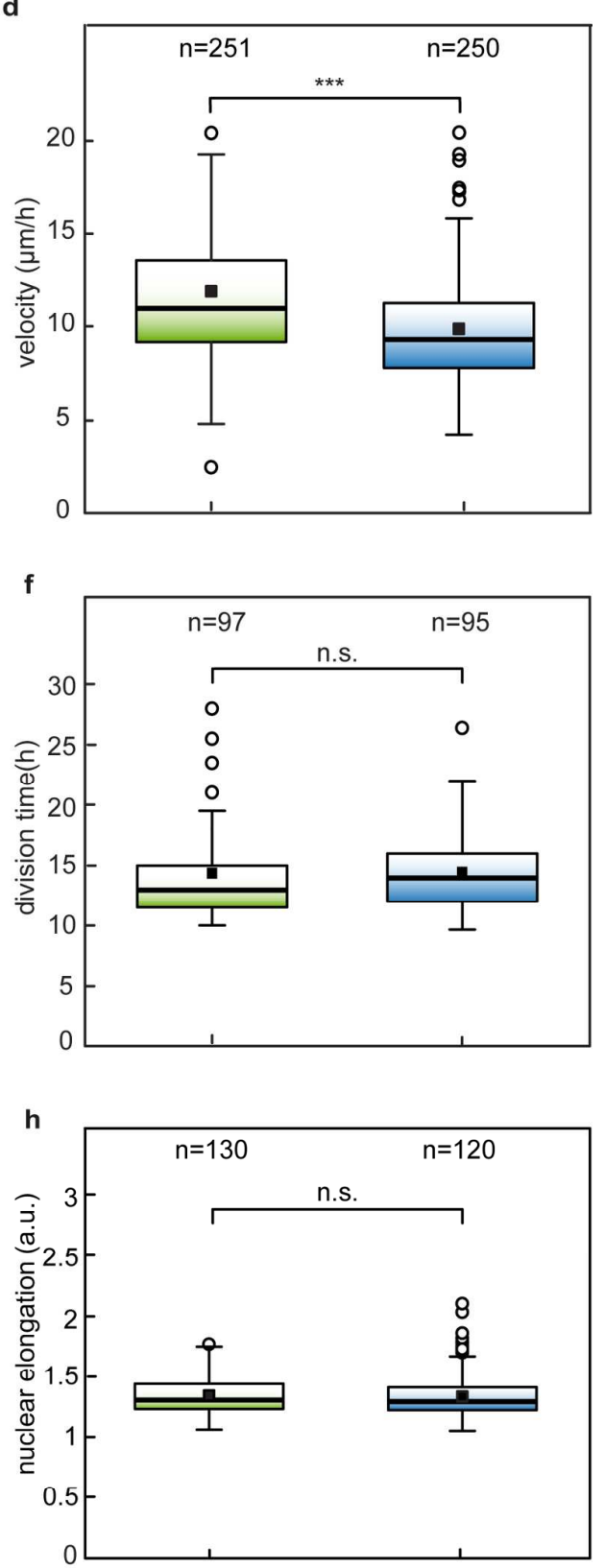
Supplementary Figure 1: Effect of gratings on migration, proliferation and nuclear deformation in HeLa Kyoto and MCF10CA1a.cll cells. Boxplots reporting the a-b) velocity, c-d) division time, e-f) nuclear elongation and $\mathbf{g - h}$ ) track orientation to the gratings (where $0^{\circ}$ corresponds to perfect alignment to the gratings), for HeLa Kyoto (left column) and MCF10CA1a.cl1 cells (right column). $(* * *$ for $\mathrm{p}<0.001, * *$ for $\mathrm{p}<0.01 *$ for $\mathrm{p}<0.1$, U-test) $\mathrm{n}$ : total number of analyzed events from 16 independent experiments. 


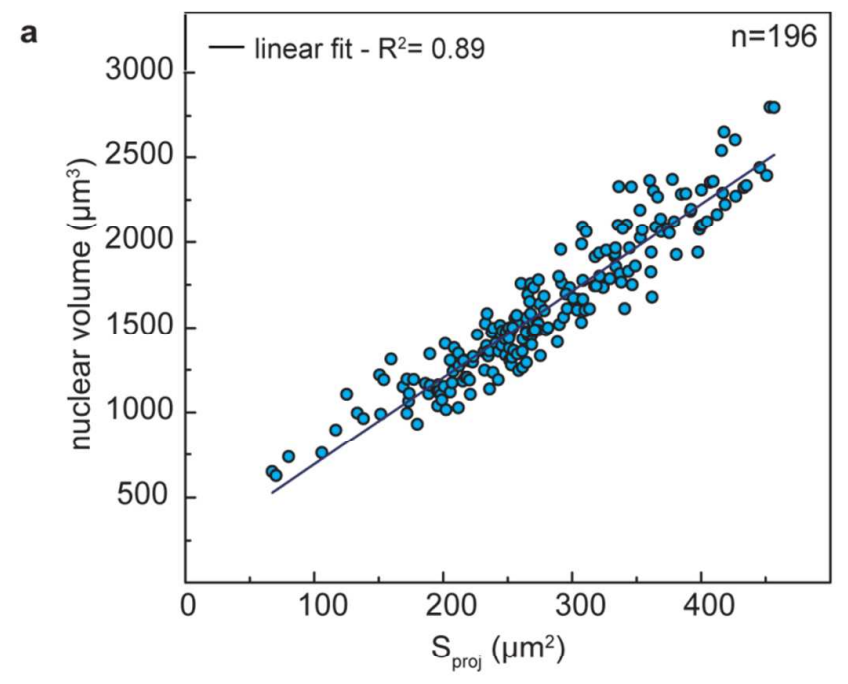

b

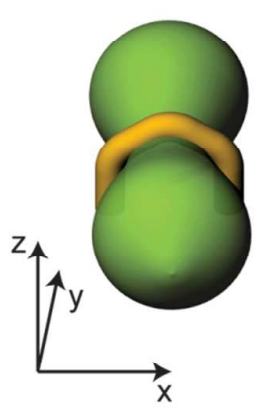

c

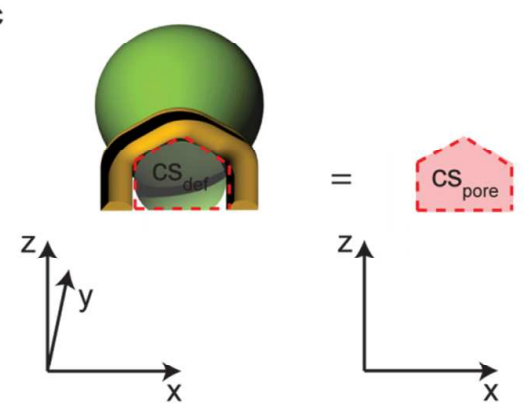

d
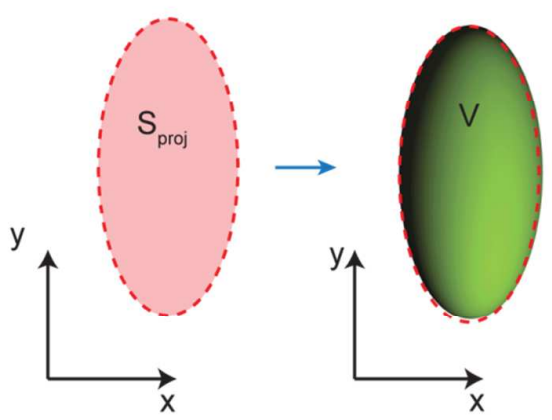

(eq.1)

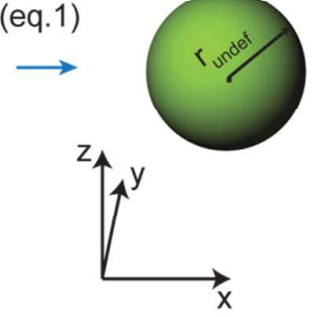

(eq.2)
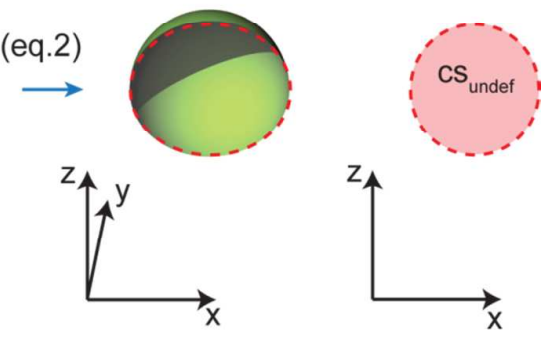

Supplementary Figure 2:: Nuclear geometry of HeLa Kyoto cells and calculation of nuclear deformation. a) Nuclear volume and corresponding projected nuclear surface $\left(S_{\text {proj }}\right)$ for individual HeLa Kyoto cells as obtained from confocal imaging and volume rendering. Data points are represented as blue circles. A linear fit $\left(\mathrm{R}^{2}=0.89\right)$ the data is reported as a black line. $\mathrm{n}$ : total number of counted cells from 3 independent experiments. b) Schematic 3D representation of a cell nucleus (green) upon the penetration of a printed pore (yellow). c) When the cross section of the pore $\left(\mathrm{CS}_{\text {pore }}\right)$ is small, the nucleus deforms assuming an hourglass shape that adapts to the pore. d) The projected nuclear surface $\left(\mathrm{S}_{\text {proj }}\right)$ is used to estimate the nuclear volume using the correlation curve reported in Supplementary Fig. 1. The estimated volume $(\mathrm{V})$ is used to calculate the radius $\left(\mathrm{r}_{\text {undef }}\right)$ and the cross section $\left(\mathrm{CS}_{\text {undef }}\right)$ of an equivalent sphere, corresponding to the undeformed nuclear state. The nuclear deformation required to penetrate a pore is then calculated from the $\mathrm{CS}_{\text {pore }} / \mathrm{CS}_{\text {undef }}$ ratio as described in eq. 1-3. 


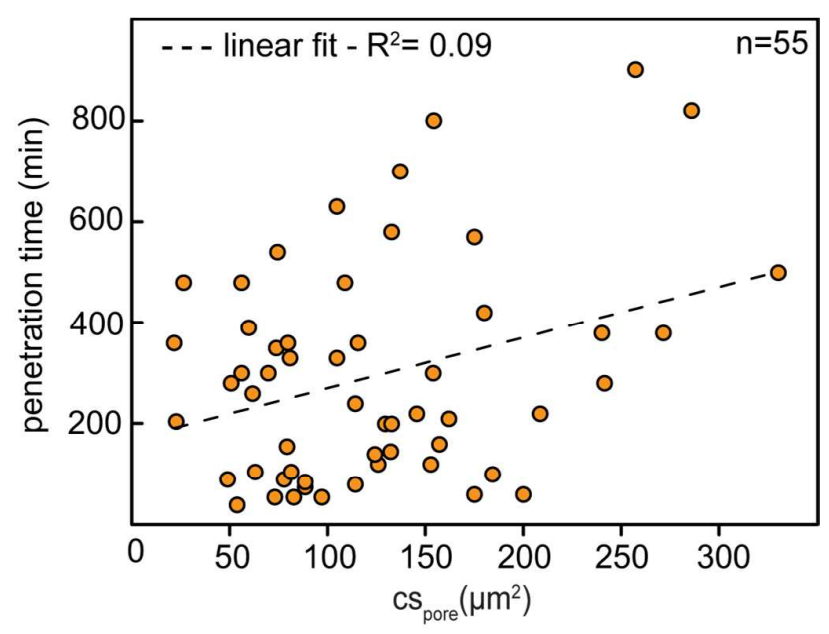

Supplementary Figure 3: $\underline{\text { Correlation between pore penetration dynamics and pore size for }}$ HeLa Kyoto cells. Individual data points are reported as solid circles. A linear fit of the data is reported as a dotted line $\left(\mathrm{R}^{2}=0.09\right)$. $\mathrm{n}$ : total number of counted cells from 16 independent experiments. 
a

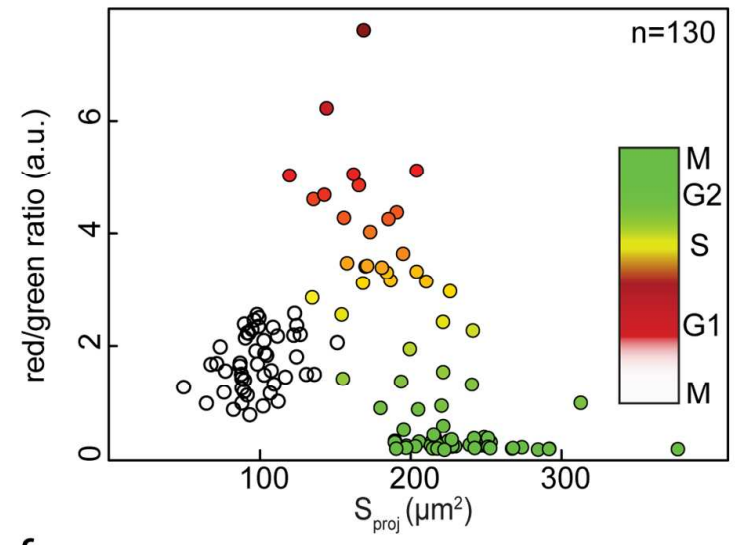

c

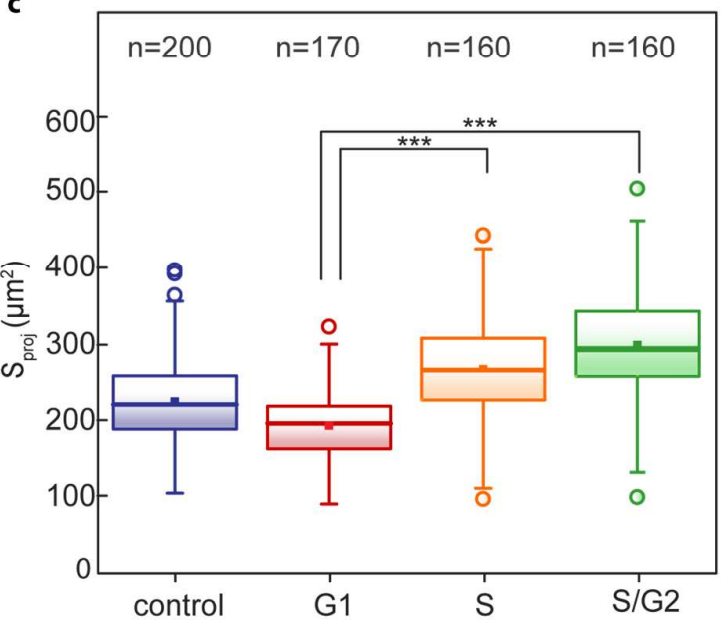

b

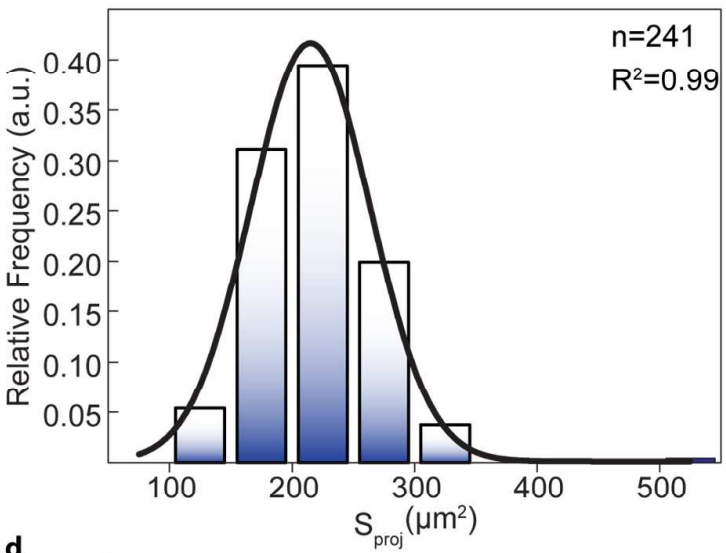

d

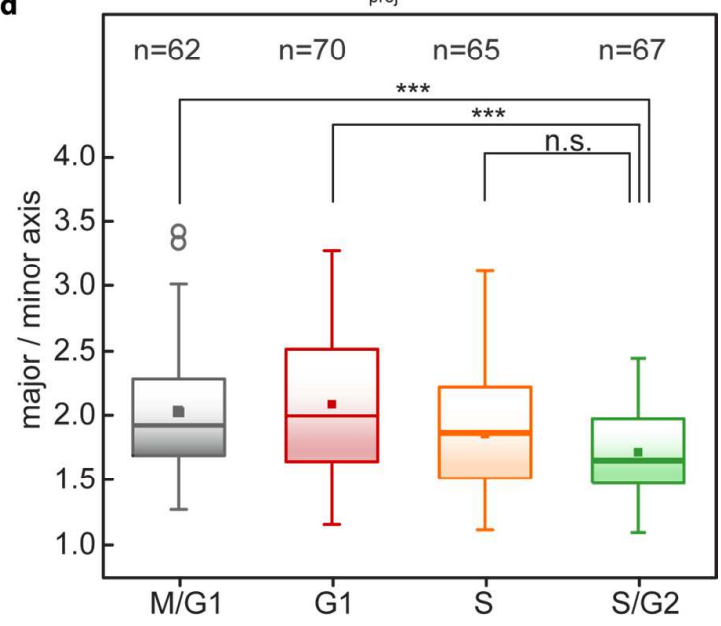

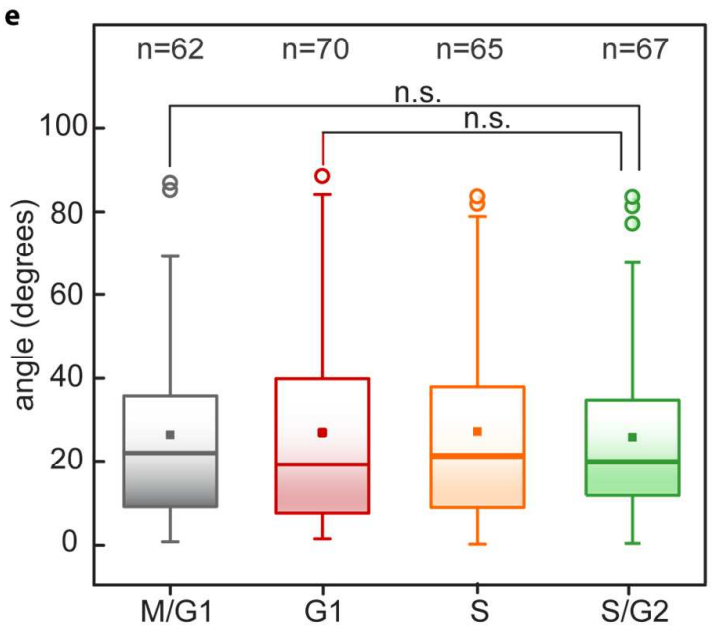

Supplementary Figure 4: Nuclear size and contact guidance as a function of the cell cycle phase in HeLa Fucci2 cells. a) Ratio of red to green fluorescence as a function of the projected nuclear surface $\left(\mathrm{S}_{\text {proj}}\right)$ for HeLa Fucci2 cells. A calibration bar (color to cell cycle phase) is reported in the right side of the panel. $\mathrm{n}$ : total number of counted cells from 3 independent experiments. b) Distribution of projected nuclear surface $\left(\mathrm{S}_{\text {proj }}\right)$ for HeLa Fucci2 cells (blue histograms). The Gaussian fit of the data (peak at 216 \pm 3.06 ) is reported as a black line n: total number of counted nuclei from 3 independent experiments. c) Projected nuclear 
surface of control HeLa Kyoto cells and of HeLa Kyoto cells arrested by chemical treatment to G1, S and G2 phases, respectively. n: total number of counted cells from 1-2 independent experiments d) Nuclear alignment to the gratings and b) nuclear elongation as a function of the cell cycle phase for HeLa Fucci2 cells. (n.s., not significant, *** for $\mathrm{p}<0.001$, ** for $\mathrm{p}<$ 0.01 , U-test) $\mathrm{n}$ : total number of counted cells from 4 independent experiments. 

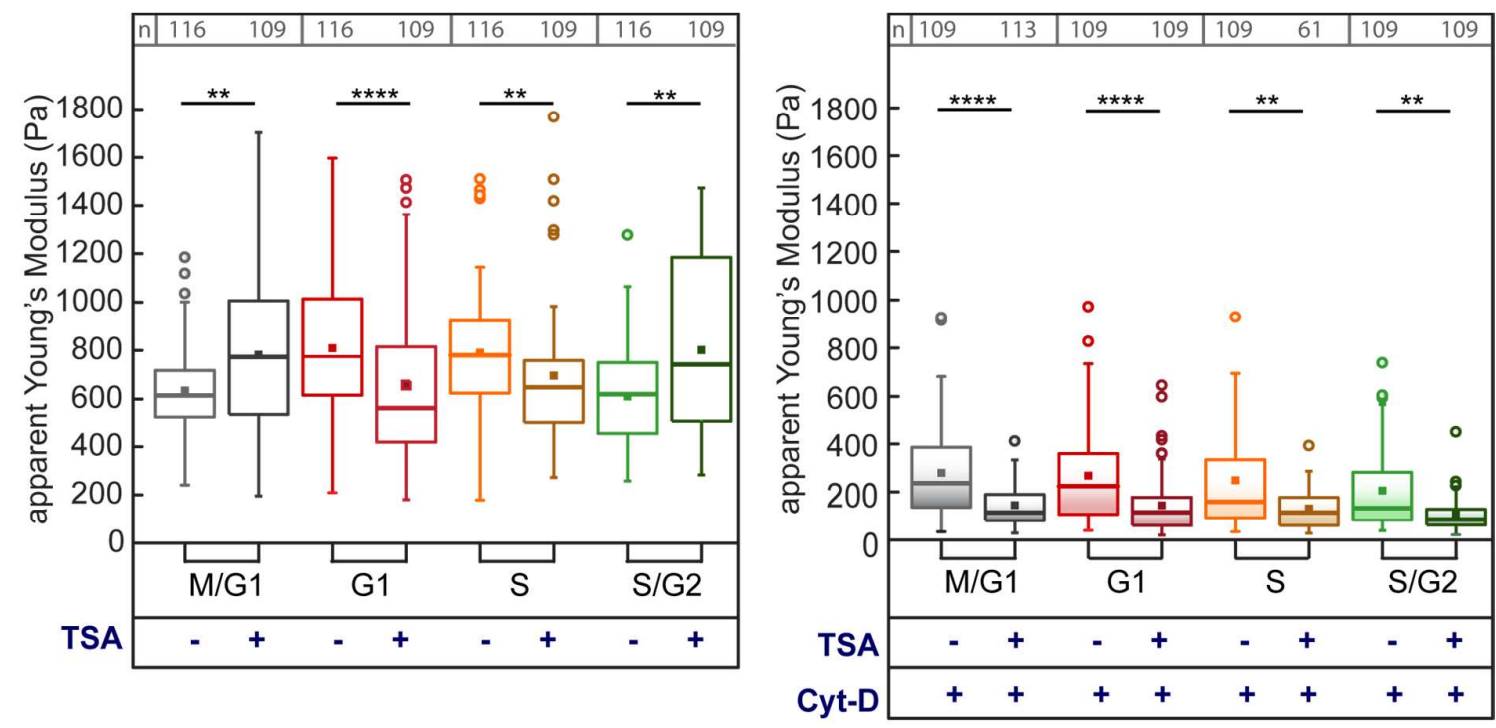

Supplementary Figure 5: Effect of Trichostatin-A on effective stiffness during cell cycle. a) Effective stiffness of the nuclear region as a function of the cell cycle phase in HeLa Fucci2 cells adhering to gratings with and without treatment with Trichostatin-A (TSA). b) Effective stiffness of the nuclear region as a function of the cell cycle phase in cells treated with Cytochalasin D and with and without treatment with Trichostatin-A. (n.s., not significant, $* * * *$ for $\mathrm{p}<0.0001, * * *$ for $\mathrm{p}<0.001, * *$ for $\mathrm{p}<0.01 \mathrm{U}$-test) $\mathrm{n}$ : total number of counted cells from 4 independent experiments. 


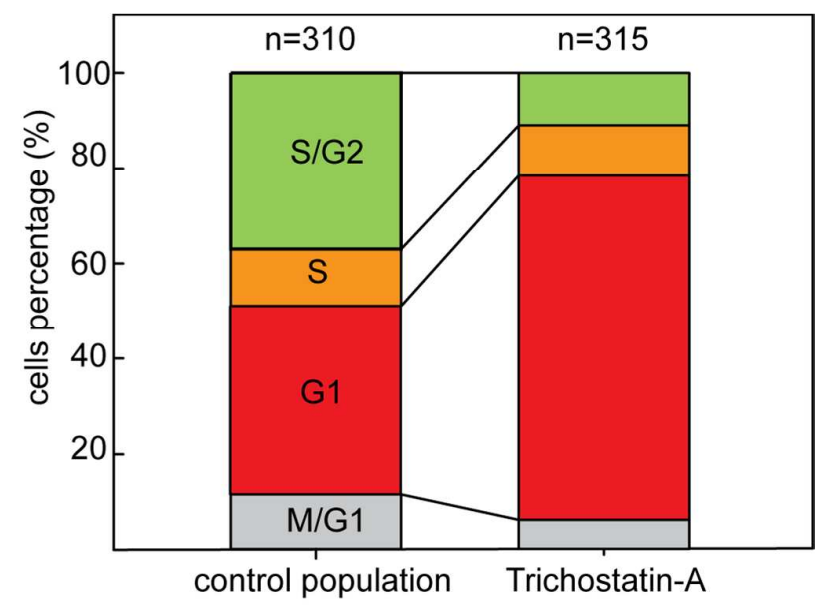

Supplementary Figure 6: Effect of Trichostatin-A on cell cycle. Prevalence of cycle phases in the control population and in the population treated with Trichostatin-A. n: total number of counted cells from 4 independent experiments.

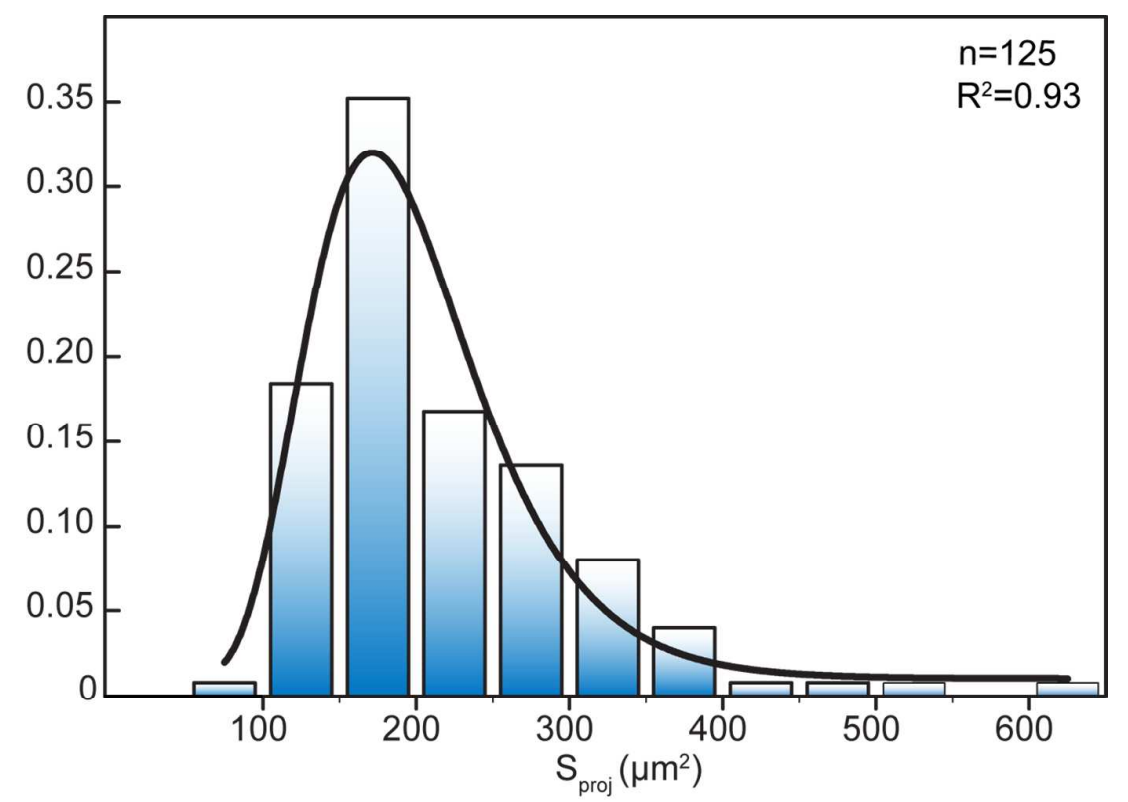

Supplementary Figure 7: Projected nuclear surface of MCF10CA1a.cll cells. Boxplots showing the distribution of projected nuclear surface $\left(\mathrm{S}_{\text {proj }}\right)$ for MCF10CA1a.cl1 cells (blue

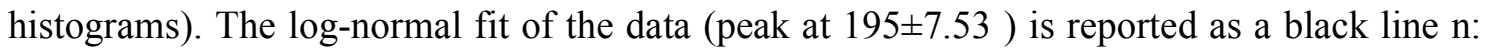
total number of counted nuclei from 5 independent experiments. 


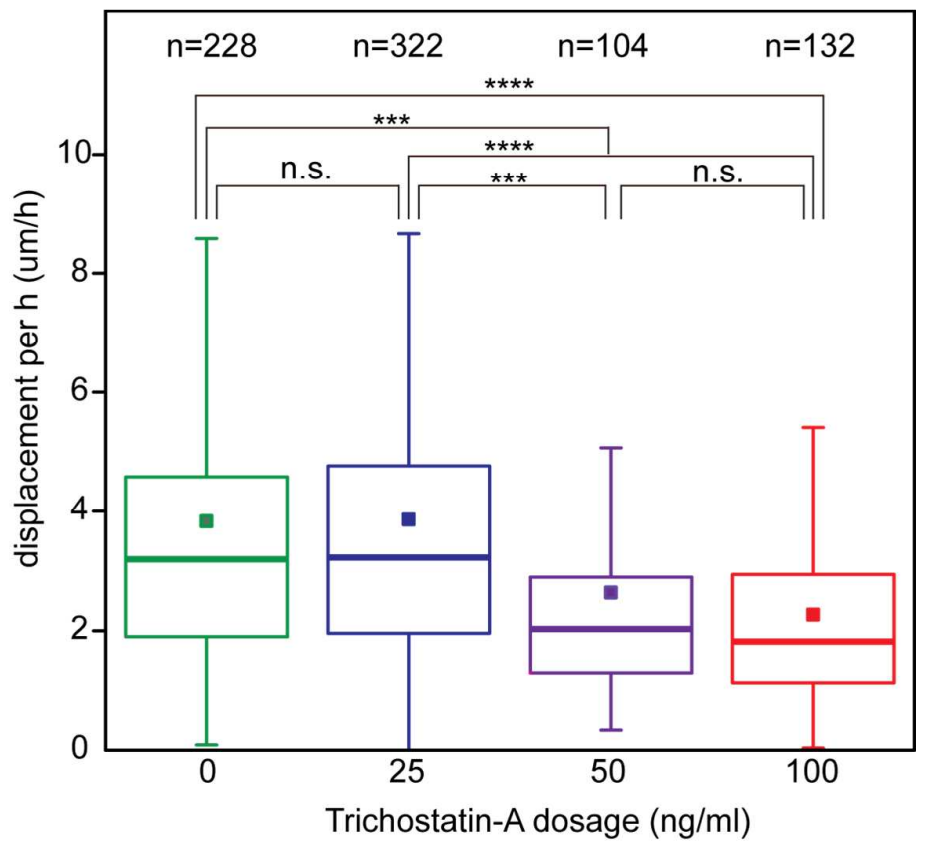

Supplementary Figure 8: Dose-dependent effect of Trichostatin-A on MCF10CA1a.cl1 migration. Boxplots showing the displacement over hour for MCF10CA1a.cll cells treated with increasing drug concentrations. (n.s., not significant, $* * * *$ for $\mathrm{p}<0.0001$, $* * *$ for $\mathrm{p}<0.001$, U-test) $\mathrm{n}$ : total number of counted cells from 3 independent experiments. 
Supplementary Video 1: Sequential nanoprinting of vertical pore arrays. Scale bar: $10 \mu \mathrm{m}$

Supplementary Video 2: Merged transmission and fluorescence time-lapse of a HeLa Kyoto cell engaging and penetrating a pore. Scale bar: $10 \mu \mathrm{m}$

Supplementary Video 3: Merged transmission and fluorescence time-lapse of a HeLa Kyoto cell engaging and failing the penetration of a pore. Scale bar: $10 \mu \mathrm{m}$

Supplementary Video 4: Merged transmission and fluorescence time-lapse of a HeLa Kyoto cell engaging a pore and dividing. The two daughter cells eventually penetrate two distinct pores. Scale bar: $10 \mu \mathrm{m}$

Supplementary Video 5: Merged transmission and fluorescence time-lapse of a HeLa Fucci2 cell engaging and penetrating a pore immediately after division. Scale bar: $10 \mu \mathrm{m}$.

Supplementary Video 6: Merged transmission and fluorescence time-lapse of a HeLa Fucci2 cell engaging and penetrating a pore in the G1 phase of the cell cycle. Scale bar: 10 $\mu \mathrm{m}$.

Supplementary Video 7: Merged transmission and fluorescence time-lapse of a HeLa Fucci2 cell treated with Trichostatin-A engaging and penetrating a pore. Scale bar: $10 \mu \mathrm{m}$.

Supplementary Video 8: Merged transmission and fluorescence time-lapse of a control HeLa Fucci2 cell engaging and penetrating a pore. Scale bar: $10 \mu \mathrm{m}$.

Supplementary Video 9: Merged transmission and fluorescence time-lapse of a MCF10CA1a.cl1 cell undergoing $88 \%$ of nuclear deformation in order to penetrate a pore. Scale bar: $10 \mu \mathrm{m}$. 


\section{$\underline{\text { Supplementary Methods }}$}

Imaging of vertical pores

The geometrical characteristics and quality of printed samples were examined by SEM with a Zeiss ULTRA 55 scanning electron microscope (FIRST Cleanroom Platform, ETH Zurich). First, an overview picture (top view) of the entire sample was acquired, and pore lines were assigned a tracking number to facilitate subsequent identification in the live microscopy experiments. Afterwards, the magnification was increased and the SEM stage was tilted in order to visualize the geometry of individual pores. The cross section of the pores was subsequently measured using the freehand selection tool of ImageJ (National Institute of Health, USA). The imaging angle of the microscope was used to convert the measured areas of the titled images to the corresponding actual pore cross section through geometric projection. The pore cross section was adjusted by changing the number of pulses during printing (Fig. 1a). The pore size variation for a given printing parameter set was $4 \%$, demonstrating the excellent control of the pore geometry offered by this platform.

\section{Cell culture}

HeLa cells were maintained in DMEM supplemented with $10 \%$ fetal bovine serum (FBS), Lglutamine, 100 units $\cdot \mathrm{ml}^{-1}$ penicillin and $100 \mu \mathrm{g} \cdot \mathrm{ml}^{-1}$ streptomycin (all from Invitrogen) at $37^{\circ} \mathrm{C}$ in a $5 \% \mathrm{CO}_{2}$ environment. Medium for HeLa Kyoto cells additionally contained 0.5 $\mathrm{g} \cdot \mathrm{ml}^{-1}$ Geneticin.

MCF10CA1a.cl1 cells ${ }^{1}$ were cultured in DMEM/F12 (1:1 ratio) containing 5\% horse serum, $1.05 \mathrm{mM} \mathrm{CaCl} 2$, and $10 \mathrm{mM}$ HEPES supplemented with $1 \%$ penicillin/streptomycin (all from Sigma). Cells were cultured at $37^{\circ} \mathrm{C}$ in $5 \%$ carbon dioxide. MCF10CA1a.cll cells stably expressing a histone H2B-GFP fusion protein were created using the Phoenix Retroviral Infection system ${ }^{2}$. Briefly, infectious retroviral particles were produced by transient Calcium phosphate-mediated transfection of Phoenix cells with a pBabe-puro-H2B-GFP plasmid. MCF10CA1a.cl1 cells were infected with the retroviral containing supernatant, followed by selection using $2 \mu \mathrm{g} / \mathrm{ml}$ Puromycin. Reagents and plasmids for retroviral infection were kindly provided by Prof. Giorgio Scita (IFOM institute, Italy). 
The printed COC substrates were sealed to the bottom of $3 \mathrm{~cm}$ culture dishes. Dishes were sterilized by overnight treatment with a solution of $80 \%$ ethanol in PBS and rinsed three times with PBS before usage. Substrates were coated with $0.01 \%$ poly-L-lysine (PLL; Sigma) according to the manufacturer's specification. Substrates were stored in PBS at $4^{\circ} \mathrm{C}$ until cell seeding. During all experimental procedures the samples were not allowed to dry, as capillary forces exert strain on the microstructures, causing damages. For the migration and penetration experiments, cells were seeded to a final confluence of $60-75 \%$ and allowed to settle for at least $6 \mathrm{~h}$ before imaging.

For Trichostatin-A treatment of HeLa Kyoto cells, $100 \mathrm{ng} / \mathrm{ml}$ of Trichostatin-A (Sigma Aldrich, USA) were added to the sample $4 \mathrm{~h}$ prior to imaging. Before exploring the effect of Trichostatin-A on MCF10CA1a.cl pore penetration efficiency, a dosage test was performed by adding 25, 50, and $100 \mathrm{ng} / \mathrm{ml}$ of Trichostatin on MCF10CA1a.cll cells and measuring cell displacement on gratings over $24 \mathrm{~h}$. The results are reported in Supplementary Figure 8 and indicate that the highest dosage of Trichostatin-A that could be added on the cells without affecting cell migration was $25 \mathrm{ng} / \mathrm{ml}$.

For the synchronization of HeLa Kyoto cells at the beginning of S-phase a double thymidine block ( $2 \mathrm{mM}$ final concentration) was performed. For arrest of HeLa Kyoto cells at G2/M phase, $10 \mu \mathrm{M}$ R03306 was added $12 \mathrm{~h}$ before imaging. For the arrest of cells at G1 phase, 20 $\mu \mathrm{M}$ Lovastatin was added to the culture medium $12 \mathrm{~h}$ before imaging.

\section{Transfection of cells with EGFP-Paxillin}

HeLa Fucci2 cells $^{3}$ were transiently transfected with EGFP-Paxillin DNA ${ }^{4}$ using JetPrime Transfection Reagent (Polyplus, BP, Illkirch, France). Cells were seeded in a 6-well plate 24 $\mathrm{h}$ before transfection, at a density of 200.000 cells/well, each of which contained $2 \mathrm{ml}$ of DMEM medium. Then cells were transfected with $2 \mu \mathrm{g}$ of EGFP-Paxillin DNA using $4 \mu \mathrm{l}$ of jetPRIME transfection reagent in $200 \mu 1$ of jetPRIME. The transfection medium was replaced after $4 \mathrm{~h}$ by fresh medium.

After $24 \mathrm{~h}$ cells were trypsinized and re-seeded on gratings into a 6 well plate. After $12 \mathrm{~h}$ the dishes were rinsed once with PBS. Cells were permeabilized and fixed by incubating in a solution of $4 \%$ paraformaldehyde with $0.2 \%$ Triton X-100 in PBS for $5 \mathrm{~min}$, followed by 
incubation in a $4 \%$ solution of PFA in PBS, all at room temperature. Dishes were rinsed gently in PBS with four changes over $5 \mathrm{~min}$. Then the gratings were removed from the 6 well plates, rinsed with DI water and mounted onto a glass slide. One drop of anti-photobleaching reagent (Fluoroshield, Sigma Aldrich) was added to the sample and then a glass coverslip was sealed onto it with nail polish. The glass coverslips were stored in the dark at $4^{\circ} \mathrm{C}$ until imaging.

\section{Scanning electron microscopy of cells}

For the SEM analysis of pore penetration, samples were washed 3 times with PBS and then incubated for $1 \mathrm{~h}$ with $2.5 \%$ glutaraldehyde in sodium cacodilate at RT. The samples were then washed 3 times with sodium cacodilate and progressively dehydrated in $50 \%, 70 \%$ and $100 \%$ ethanol. The specimens were stored in ethanol, until they were dried using a critical point dryer (BAL-TEC CPD 030). The specimens were sputter-coated with gold/palladium using a BAL-TEC SCD-050 sputter coater and imaged with a Zeiss ULTRA 55 scanning electron microscope.

Quantifying effective cell stiffness and nuclear stiffness by atomic force microscopy (AFM) indentation measurements

For AFM indentation measurements, a Nanowizard I with a Cellhesion module (JPK Instruments, Berlin, Germany) was used. The AFM was mounted on top of an inverted light microscope equipped with epifluorescence to visualize red and green fluorescence of HelaFucci2 cells. Arrow-T1 cantilevers (Nanoworld, Neuchatel, Switzerland) were modified with a polystyrene bead (radius $5 \mu \mathrm{m}$, microparticles $\mathrm{GmbH}$, Berlin, Germany) with the aid of epoxy glue to obtain a well-defined indenter geometry and decrease local strain during indentation. Cantilevers were calibrated prior experiments using built-in procedures of the AFM data analysis software (JPK Instruments). A heat-controlled chamber (Petri dishHeater, JPK Instruments) was used to perform experiments at $37^{\circ} \mathrm{C}$. Cells were seeded $48 \mathrm{~h}$ before the experiment at 70.000 cells/well onto poly-L-lysine coated COC films that had been glued onto the bottom of round (diameter $35 \mathrm{~mm}$ ) Petri dishes (FluoroDishTM, WPI, Sarasota, US). Experiments were conducted in $\mathrm{CO}_{2}$ independent medium (Life Technologies). During the AFM indentation experiment, a cell of a particular interphase stage 
was selected based on its epifluorescence signal. Then the bead was lowered at a speed of 5 $\mu \mathrm{m} / \mathrm{sec}$ onto the cell surface over the nucleus. After reaching the set point of $7 \mathrm{nN}$, the cantilever was retracted. During the force-distance cycle, the force over piezo position was recorded. The resulting force-distance curves were transformed into force- versus- tip sample separation curves $^{5}$ and fitted with the Hertz/Sneddon model for a spherical indenter ${ }^{6,7}$ using the JPK analysis software (JPK DP, JPK Instruments). A Poisson ratio of 0.5 was used for the calculation of the apparent Young's modulus. An indentation depth of 1.5-2 $\mu \mathrm{m}$ was used for analysis. For Trichostatin-A treatment, cells were pre-incubated for 12-14 h with $200 \mathrm{ng} / \mathrm{ml}$ Trichostatin -A. In experiments, where actin cytoskeleton was perturbed, $\mathrm{CO}_{2}$-independent medium with $600 \mathrm{nM}$ Cytochalasin D (Sigma-Aldrich) was added for $30 \mathrm{~min}$ onto the cells. Cells were then probed in presence of Cytochalasin D. Control cells were probed in $\mathrm{CO}_{2}$ independent medium with equivalent volume of DMSO (vehicle control).

\section{Image analysis}

For the 3D rendering of cell nuclei, HeLa Kyoto cells or MCF10CA1a.cll cells were cultured on gratings and fluorescent Z-stacks of the green signal emitted by the cell nuclei $(\Delta \mathrm{Z}=0.1$ $\mu \mathrm{m})$ were collected using a Nikon-Ti spinning disk confocal microscope (Nikon, Japan) equipped with an Andor DU-888 camera (Oxford Instruments, United Kingdom) and an incubation chamber (Life Imaging Services, Switzerland), where temperature, $\mathrm{CO}_{2}$ concentration, and humidity were maintained at $37^{\circ} \mathrm{C}, 5 \%$, and $95 \%$ respectively. Images were collected with a $60 \mathrm{X}$ objective (Apo 60x Oil $\lambda \mathrm{S}$ DIC N2). The resulting stacks were loaded in Imaris 8.0.1 and a scene was created in the program. Rendering of individual cell nuclei was obtained applying an automatically-detected image intensity threshold based on the algorithm developed by Ridler and Calvard ${ }^{8}$. The surface and volume for the rendered objects were then obtained using the measurement tool of Imaris. These data were used to obtain a linear correlation between the projected area and the volume of individual cell nuclei (Supplementary Fig. 1). 


\section{References}

1. Tang, Z.; Yu, M.; Miller, F.; Berk, R. S.; Tromp, G.; Kosir, M. A. Increased Invasion through Basement Membrane by Cxcl7-Transfected Breast Cells. Am. J. Surg. 2008, 196, 690-696.

2. Swift, S.; Lorens, J.; Achacoso, P.; Nolan, G. P., Rapid Production of Retroviruses for Efficient Gene Delivery to Mammalian Cells Using 293t Cell-Based Systems. In Current Protocols in Immunology, John Wiley \& Sons, Inc.: 2001.

3. Sakaue-Sawano, A.; Kobayashi, T.; Ohtawa, K.; Miyawaki, A. Drug-Induced Cell Cycle Modulation Leading to Cell-Cycle Arrest, Nuclear Mis-Segregation, or Endoreplication. BMC Cell Biol. 2011, 12, 1-12.

4. Kubow, K. E.; Horwitz, A. R. Reducing Background Fluorescence Reveals Adhesions in 3d Matrices. Nat. Cell Biol. 2011, 13, 3-7.

5. Radmacher, M. Measuring the Elastic Properties of Living Cells by the Atomic Force Microscope. Atomic Force Microscopy in Cell Biology 2002, 68, 67-90.

6. Sneddon, I. N. The Relation between Load and Penetration in the Axisymmetric Boussinesq Problem for a Punch of Arbitrary Profile. Int. J. Eng. Sci. 1965, 3, 47-57.

7. Heinrich, H. Ueber Die Beruehrung Fester Elastischer Koerper. Journal für die reine und angewandte Mathematik 1881, 156-171.

8. Ridler, T.; Calvard, S. Picture Thresholding Using an Iterative Selection Method. IEEE transactions on Systems, Man and Cybernetics 1978, 8, 630-632. 